\title{
Relación entre Síntomas de Trastornos Témporo- mandibulares Auto-Reportados y el Movimiento Mandibular de Sujetos Adultos Jóvenes
}

\author{
Relationship Between Symptoms of Self-Reported Temporomandibular \\ Disorders and Mandibular Movement of Young Adult Subjects
}

Alain Arias 1, 2, 4; Diego Saravia²; Melanie Quintana ${ }^{3}$; Fernando Dias ${ }^{1,2}$; María Florencia Lezcano1,2; Constanza Farfán² \& Ramón Fuentes ${ }^{1,2}$

\begin{abstract}
ARIAS, A.; SARAVIA, D.; QUINTANA, M.; DIAS, F.; LEZCANO, M. F.; FARFÁN, C. \& FUENTES, R. Relación entre síntomas de trastornos témporomandibulares auto-reportados y el movimiento mandibular de sujetos adultos jóvenes. Int. J. Odontostomat., 13(4):466-474, 2019.

RESUMEN: Tanto la limitación del movimiento mandibular como el auto reporte de síntomas de trastornos témporomandibulares (TTM) son utilizados como elementos para el diagnóstico de pacientes en la clínica. Sin embargo, la relación entre la presencia de síntomas y el movimiento mandibular no está del todo clara. Por ello, el objetivo de este estudio fue relacionar la presencia de síntomas asociados a TTM con el movimiento mandibular en una muestra de sujetos adultos jóvenes y sanos. En este estudio exploratorio participaron 40 estudiantes de Odontología, médicamente sanos, con arco dentario maxilar y mandibular continuo y completo (hasta el primer molar). A cada uno se les solicitó responder un cuestionario de auto-reporte de síntomas asociados a TTM recomendado por la American Academy of Orofacial Pain (AAOP). También se realizó un registro y análisis de sus movimientos mandibulares utilizando articulografía electromagnética 3D, con el objetivo de correlacionar la presencia de síntomas con el rango y trayectoria de apertura, y el área comprendida en lo que se describe como polígono de Posselt en el plano frontal y sagital. No se encontraron diferencias significativas entre las respuestas a cada pregunta entre hombres y mujeres. De acuerdo a las respuestas y según Delcanho (1994), 12 participantes resultaron necesitar una evaluación más exhaustiva. En este grupo se encontró que el área del polígono de Posselt en el plano frontal, fue significativamente menor que el de aquellos participantes que no requieren una evaluación más exhaustiva $(p=0,003)$. Los valores de la trayectoria de apertura mandibular varían según de que síntoma se trate. Los principales síntomas que afectarían los valores de los movimientos bordeantes son la "dificultad para abrir la boca" y el "ruido en las articulaciones mandibulares". Con este estudio, hemos puesto de manifiesto que la limitación del movimiento mandibular como signo de TTM debe evaluarse con cautela, debido a que según la presencia de determinados síntomas y otras características de los sujetos (como el sexo), su valor podría variar.
\end{abstract}

PALABRAS CLAVE: trastornos temporomandibulares, movimiento mandibular, articulografía electromagnética.

INTRODUCCIÓN

Los trastornos témporomandibulares (TTM) son una de las principales causas de dolor no dental en la región orofacial y son definidos por la Academia Americana de Dolor Orofacial (AAOP) como un término colectivo que abarca numerosos problemas clínicos que afectan a los músculos masticadores, la articulación témporomandibular (ATM) (Klasser \& Greene,
2009) y que, en cierto nivel, también podrían afectar al propio funcionamiento de la articulación y, por tanto, a la dinámica mandibular. La dinámica mandibular se define como los movimientos que la mandíbula realiza en los tres ejes del espacio mediante la combinación de movimientos de traslación y rotación condilar (Manns Freese, 2011). Los músculos de la

\footnotetext{
${ }^{1}$ Department of Integral Adults Dentistry, Dental School, Universidad de La Frontera, Temuco, Chile.

${ }^{2}$ Research Centre for Dental Sciences (CICO), Dental School, Universidad de La Frontera, Temuco, Chile.

${ }^{3}$ Undergraduate student, Dental School, Universidad de La Frontera, Temuco, Chile

${ }^{4}$ Universidad Adventista de Chile, Chillán, Chile.
} 
ARIAS, A.; SARAVIA, D.; QUINTANA, M.; DIAS, F.; LEZCANO, M. F.; FARFÁN, C. \& FUENTES, R. Relación entre síntomas de trastornos témporomandibulares auto-reportados y el movimiento mandibular de sujetos adultos jóvenes. Int. J. Odontostomat., 13(4):466-474, 2019.

masticación posibilitan los movimientos mientras que las ATM determinan el patrón de los mismos (Granger, 1954). Los síntomas asociados a la presencia de TTM son diversos y varían de acuerdo a su localización e intensidad. Estos incluyen, principalmente, dolor en los músculos de la masticación, sensación de fatiga y tensión muscular, disminución de la amplitud de los movimientos mandibulares, artralgia y ruido articular (Taboada et al., 2004). El dolor muscular puede no percibirse en el sitio de la lesión y suele expresarse como síntomas óticos, artralgia y cefalea, entre otros (Ramirez Aristeguieta et al., 2004). Dependiendo de la severidad de la sintomatología, los TTM pueden llegar a obstaculizar seriamente la calidad de vida de una persona.

La literatura señala que en adolescentes de Alemania y China se ha encontrado una prevalencia de TTM cercana al $13 \%$ (Wu \& Hirsch, 2010). En Latinoamérica, la prevalencia reportada varía desde el $6 \%$ (Pereira et al., 2009) hasta el $46 \%$ (CasanovaRosado et al., 2006). En un estudio realizado en Estados Unidos se observó que la prevalencia de TTM diagnosticados en adolescentes fue de $26,88 \%$, siendo el diagnóstico más común el dolor miofacial con una prevalencia de 8,06 \% (Manfredini et al., 2011). Asimismo, la mayor prevalencia para el diagnóstico de TTM se encuentra en el rango de los 30 a 45 años de edad (Aravena et al., 2016). Se destaca que en el sexo femenino las parafunciones y la ansiedad juegan un papel relevante estando fuertemente asociadas con la presencia de síntomas y signos clínicos de TTM (Paulino et al., 2018). Esto coincide con el reporte de que en general las mujeres sufren con más frecuencia de dolor crónico, relatando un dolor de mayor intensidad y duración que el de los hombres (Sena et al., 2013). Resulta interesante analizar estos hallazgos teniendo en consideración que el rango de apertura mandibular en mujeres es significativamente menor al de los hombres. Así lo dejan en claro algunos estudios como el de Li et al. (2017) realizado en China donde el promedio del rango de apertura máxima en hombres fue de $54,18 \pm 5,21 \mathrm{~mm}$, mientras que en mujeres fue de 49,62 $\pm 3,69 \mathrm{~mm}$, o el estudio de AlDlaigan \& Asiry (2014) realizado en Arabia Saudita donde el rango de apertura máxima en hombres fue de $43,5 \pm 4,23 \mathrm{~mm}$, mientras que en las mujeres fue de $35,5 \pm 4,4 \mathrm{~mm}$.

En vista de lo anterior, el objetivo de este estudio explorativo fue relacionar la presencia de síntomas asociados a TTM con los rangos de movimiento mandibular en una muestra de sujetos adultos jóve- nes y sanos. Para ello se utilizó un cuestionario de auto-reporte que permite recopilar en forma breve y sencilla información concerniente a la presencia actual o pasada de síntomas asociados a TTM. La presencia o ausencia de síntomas fue relacionada con mediciones altamente precisas del movimiento mandibular, realizadas con articulografía electromagnética en tres dimensiones.

\section{MATERIAL Y MÉTODO}

Se realizó un estudio exploratorio, observacional y de corte transversal que contó con la aprobación del Comité Ético Científico (CEC) de la Universidad de La Frontera ( $\mathrm{N}^{\circ}$ de protocolo 069/15). En este estudio se invitó a participar a estudiantes de la Facultad de Odontología de la Universidad de La Frontera que no estuvieran bajo tratamiento médico u odontológico, previa firma del respectivo consentimiento informado. Se incluyeron participantes jóvenes mayores de 18 años, hombres y mujeres, médicamente sanos, con arco dentario maxilar y mandibular contínuo y completo (hasta el primer molar), con normoclusión y sin aparatos de ortodoncia. A cada participante se les explicó el objetivo del estudio y se les solicitó completar un cuestionario de auto-reporte para síntomas de TTM (Delcanho) que es parte de un examen de screening recomendado por American Academy of Orofacial Pain (Tabla I). El cuestionario consiste en 10 preguntas con respuestas dicotómicas (Si/No) y evalúa la presencia de síntomas asociados a TTM. De acuerdo a Delcanho y McNeill (1993), si un paciente responde afirmativamente a cualquiera de las preguntas de la 1 a la 3 del cuestionario, se considera que el paciente necesita un examen más exhaustivo ya que es probable que presente un TTM (screening de TTM), mientras que si responden afirmativamente las preguntas del 4 al 10, se considera que representan síntomas que requieren una evaluación más exhaustiva por parte de un especialista.

Para el registro y análisis de los movimientos mandibulares se utilizó articulografía electromagnética 3D (EMA 3D, articulógrafo AG501, Carstens Medizinelektronik, Bovenden, Germany). Para ello, se utilizaron 4 sensores colocados en puntos específicos de la cabeza del participante tal y como se describe en Fuentes et al. (2018). En resumen, se utilizaron tres sensores del articulógrafo en el punto cutáneo del proceso mastoides derecho $\left(1^{\circ}\right)$ e izquierdo $\left(2^{\circ}\right)$, por detrás de cada oreja, y en la glabela $\left(3^{\circ}\right)$. Un cuarto 
Tabla I. Cuestionario de auto-reporte para síntomas de TTM, recomendado por la American Academy Pain (AAOP).

\begin{tabular}{cl}
\hline$N^{\circ}$ & \multicolumn{1}{c}{ Preguntas de screening } \\
\hline 1 & ¿Tiene usted dificultad o dolor al abrir su boca, por ejemplo al bostezar? \\
2 & ¿Siente que su mandíbula se le "atasca", se le "traba" o se le "sale"? \\
3 & ¿Tiene dificultad o dolor cuando mastica, habla o usa sus mandíbulas? \\
4 & ¿Ha notado ruidos en las articulaciones mandibulares? \\
5 & ¿Siente regularmente sus mandíbulas rígidas, apretadas (agarrotadas), cansadas? \\
6 & ¿Tiene dolor en/o alrededor de los oídos, las sie nes o las mejillas? \\
7 & ¿Tiene frecuentes dolores de cabeza o dolor de cuello o de nuca? \\
8 & ¿Ha tenido una lesión o traumatismo reciente en su cabeza, cuello o mandíbula? \\
9 & ¿Ha notado o senti do algún cambio reciente en su mordida? \\
10 & ¿Ha sido tratado anteriormente por un problema de la articulación témporomandibular?
\end{tabular}

$\left(4^{\circ}\right)$ sensor fue colocado en la línea media interincisiva mandibular (en la papila interdentaria o encía libre). De esta forma, los tres primeros sensores actúan como un sistema de referencia para el registro del movimiento del cuarto sensor puesto en la mandíbula, lo que permite descartar el movimiento natural de la cabeza del participante y registrar solo el movimiento relativo de la mandíbula, con un alto grado de precisión espacial y temporal.

Luego, cada participante fue sentado bajo las bobinas transmisoras del articulógrafo en una posición cómoda y erguida, con la vista al frente y el plano oclusal paralelo a la horizontal. A continuación se les solicitó que realizaran una serie de movimientos mandibulares desde la posición de máxima intercuspidación (PMI), tal y como se describe en Fuentes et al. Los movimientos realizados fueron: máxima apertura, máxima lateralidad sin contacto dentario (de- recha e izquierda) (Fig. 1A), máxima lateralidad con contacto dentario (derecho e izquierdo), máxima apertura bordeante lateral (derecha e izquierda) (Fig. 1B), máxima protrusión contactante, máxima apertura en protrusión, retrusión máxima contactante y máxima apertura en retrusión (Fig. 1C). De esta forma fue posible describir el llamado "polígono de Posselt" en el plano frontal y sagital del espacio. Cada movimiento se repitió y grabó tres veces para así obtener un promedio de sus medidas cuantitativas.

Una vez completado el registro de los movimientos anteriores, los datos de posición obtenidos con el articulógrafo fueron exportados y procesados con el software MATLAB (MathWorks, Inc., USA) mediante rutinas de cálculo o scripts especialmente diseñados para este estudio. De este modo se obtuvo el rango (valor del desplazamiento entre el punto de PMl y el de apertura máxima, en el eje vertical) y trayectoria
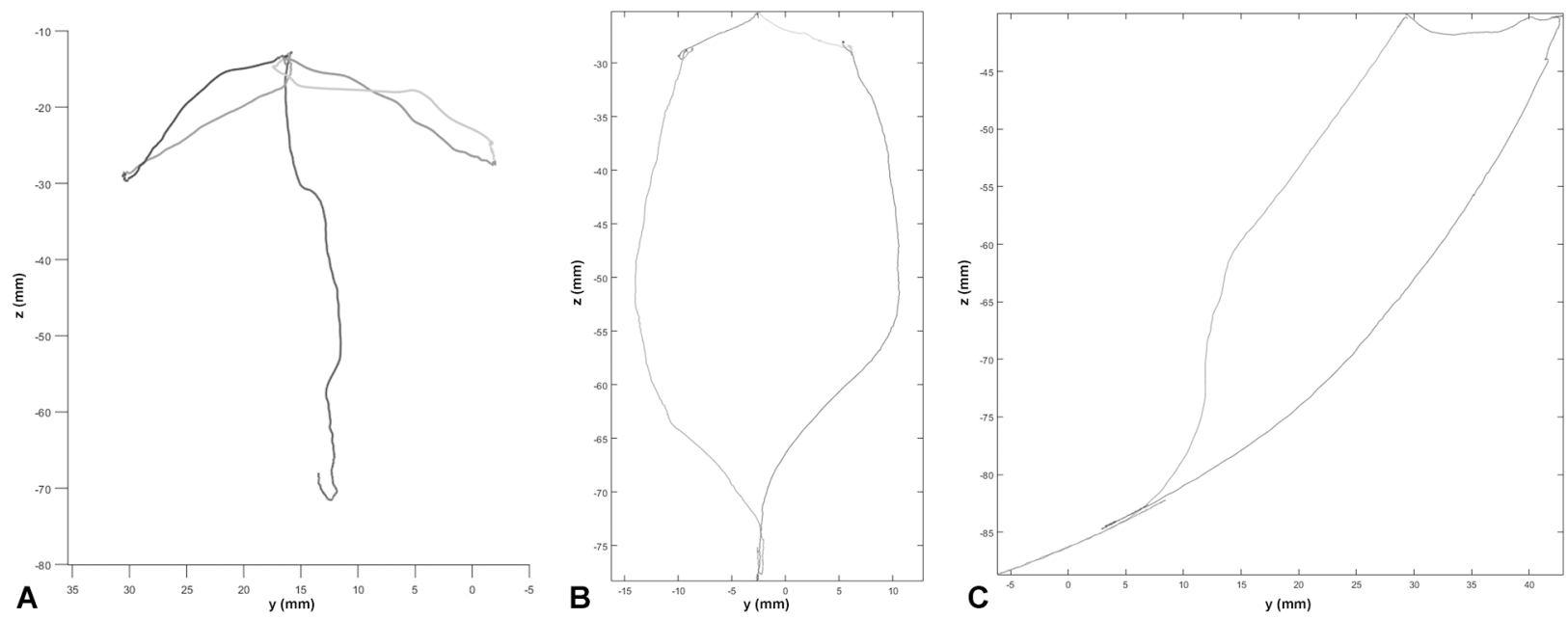

Fig. 1. Gráficos representativos del movimiento mandibular registrado en cada participante. A) Máxima apertura y máxima lateralidad sin contacto dentario (derecha e izquierda). B) Máxima lateralidad con contacto dentario (derecho e izquierdo) y máxima apertura bordeante lateral (derecha e izquierda); conformando el denominado Polígono de Posselt en el plano frontal. C) Máxima protrusión contactante, máxima apertura en protrusión, retrusión máxima contactante y máxima apertura en retrusión; conformando el denominado Polígono de Posselt en el plano sagital. 
ARIAS, A.; SARAVIA, D.; QUINTANA, M.; DIAS, F.; LEZCANO, M. F.; FARFÁN, C. \& FUENTES, R. Relación entre síntomas de trastornos témporomandibulares auto-reportados y el movimiento mandibular de sujetos adultos jóvenes. Int. J. Odontostomat., 13(4):466-474, 2019.

(valor del recorrido que realiza la mandíbula durante el trayecto entre PMI y el punto de apertura máxima) de la apertura máxima y las áreas de los polígonos de Posselt en el plano frontal y sagital (que se describen mediante el resto de movimientos mandibulares registrados). Los datos cuantitativos del movimiento mandibular fueron comparados entre participantes agrupados según sexo y tipo de respuestas a las preguntas del cuestionario (hombres que respondieron "sí", hombres que respondieron "no", mujeres que respondieron "si", mujeres que respondieron "no"). Además, los participantes fueron clasificados según sus respuestas en "sí requieren" o "no requieren" una evaluación y documentación más exhaustiva para la búsqueda y diagnóstico de TTM (Delcanho).

El análisis estadístico se realizó con el software SigmaPlot 12.0 (Systat Software Inc., San Jose, CA, USA). Se utilizó la prueba de Shapiro - Wilk para determinar la normalidad de los datos. Para la presentación de datos cuantitativos se utilizó la media \pm desviación estándar y se aplicó ANOVA de un factor seguido del post-test de Holm-Sidak / Kruskal-Wallis para el contraste de variables. Se utilizó un valor $p<0,05$ como umbral de significancia.

\section{RESULTADOS}

Se evaluaron a 40 participantes de los cuales 21 eran mujeres y 19 eran hombres, con una edad promedio de 19,78 $\pm 2,21$ años $(19,72 \pm 2,24$ años para las mujeres y 19,72 $\pm 2,21$ años para los hombres). El $85 \%(n=34)$ de los participantes reportó la presencia de al menos un síntoma (respuesta afirmativa al menos a una pregunta), mientras que el $15 \%$ $(n=6)$ restante no reporto ninguno. La distribución del auto reporte de síntomas asociados a TTM de los par- ticipantes agrupados por sexo se describe en la Tabla II. La media y la desviación estándar de los datos cuantitativos del movimiento mandibular de los participantes agrupados por sexo se presentan en Tabla III.

No se encontraron diferencias significativas entre el tipo de respuesta otorgadas por hombres y mujeres para cada pregunta del cuestionario. Para las preguntas 8 ( $¿ H a$ tenido una lesión o traumatismo reciente en su cabeza, cuello o mandíbula?) y 9 ( ¿Ha notado o sentido algún cambio reciente en su mordida?) todos los participantes respondieron "no" presentar el síntoma referido. De acuerdo a Delcanho, los participantes de este estudio fueron clasificados según la cantidad de respuestas afirmativas a las preguntas y al tipo de preguntas del cuestionario, en si requieren o no una evaluación más exhaustiva para la búsqueda de algún trastorno témporomandibular. De esta forma, solo 12 participantes resultaron necesitar una evaluación más exhaustiva, razón por la cual fueron derivados a un especialista. En este grupo de participantes se encontró que el área del polígono de Posselt en el plano frontal que describieron $\left(381,21 \pm 111,12 \mathrm{~mm}^{2}\right)$, fue significativamente menor al del otro grupo de participantes $(470,91 \pm 160,85$ $\mathrm{mm}^{2}$ ), que no requieren una evaluación más exhaustiva ( $p=0,003$; Fig. $2 C$ ). En el análisis del rango y trayectoria de apertura, y área del polígono de Posselt en el plano sagital, no se encontraron diferencias significativas entre estos dos grupos de participantes ( $p=0,505, p=0,624$ y $p=0,322$, respectivamente; Fig. $2 A, B$ y $D)$.

Al comparar los valores del rango de apertura y área del polígono de Posselt en el plano sagital entre los participantes agrupados según sexo y tipo de respuestas (afirmativas o negativas), no se encontraron diferencias significativas para ninguna de las preguntas del cuestionario.

Tabla II. Distribución del número de respuestas afirmativa («sí») o negativa («no») a cada una de las preguntas del cuestionario, agrupadas según el sexo de los participantes.

\begin{tabular}{|c|c|c|c|c|c|}
\hline & \multicolumn{2}{|c|}{ Mujer } & \multicolumn{2}{|c|}{ Hombre } & \multirow{2}{*}{$p$-value } \\
\hline & Respuesta "sí" & Respuesta "no" & Respuesta "sí" & Respuesta "no" & \\
\hline Pregunta 1 & 4 & 17 & 4 & 15 & 0,874 \\
\hline Pregunta 2 & 5 & 16 & 5 & 14 & 0,855 \\
\hline Pregunta 3 & 5 & 16 & 2 & 17 & 0,270 \\
\hline Pregunta 4 & 12 & 9 & 14 & 5 & 0,273 \\
\hline Pregunta 5 & 5 & 16 & 3 & 16 & 0,527 \\
\hline Pregunta 6 & 5 & 16 & 5 & 14 & 0,855 \\
\hline Pregunta 7 & 10 & 11 & 9 & 10 & 0,987 \\
\hline Pregunta 8 & 0 & 21 & 0 & 19 & - \\
\hline Pregunta 9 & 0 & 21 & 0 & 19 & - \\
\hline Pregunta 10 & 1 & 20 & 1 & 18 & 0,942 \\
\hline
\end{tabular}


Tabla III. Promedio \pm desviación estándar del movimiento mandibular según sexo de los participantes.

\begin{tabular}{lcccc}
\hline Sexo & Rango Apertura $(\mathrm{mm})$ & $\begin{array}{c}\text { Trayectoria Apertura } \\
(\mathrm{mm})\end{array}$ & $\begin{array}{c}\text { Área Polígono Posselt } \\
\text { Frontal }\left(\mathrm{mm}^{2}\right)\end{array}$ & $\begin{array}{c}\text { Área Polígono Posselt } \\
\text { Sagital }\left(\mathrm{mm}^{2}\right)\end{array}$ \\
\hline Mujer & $41,60 \pm 5,42$ & $60,95 \pm 5,98$ & $457,79 \pm 157,43$ & $73,05 \pm 38,38$ \\
Hombre & $42,59 \pm 6,08$ & $66,38 \pm 10,97$ & $428,76 \pm 137,09$ & $70,41 \pm 39,98$ \\
\hline
\end{tabular}
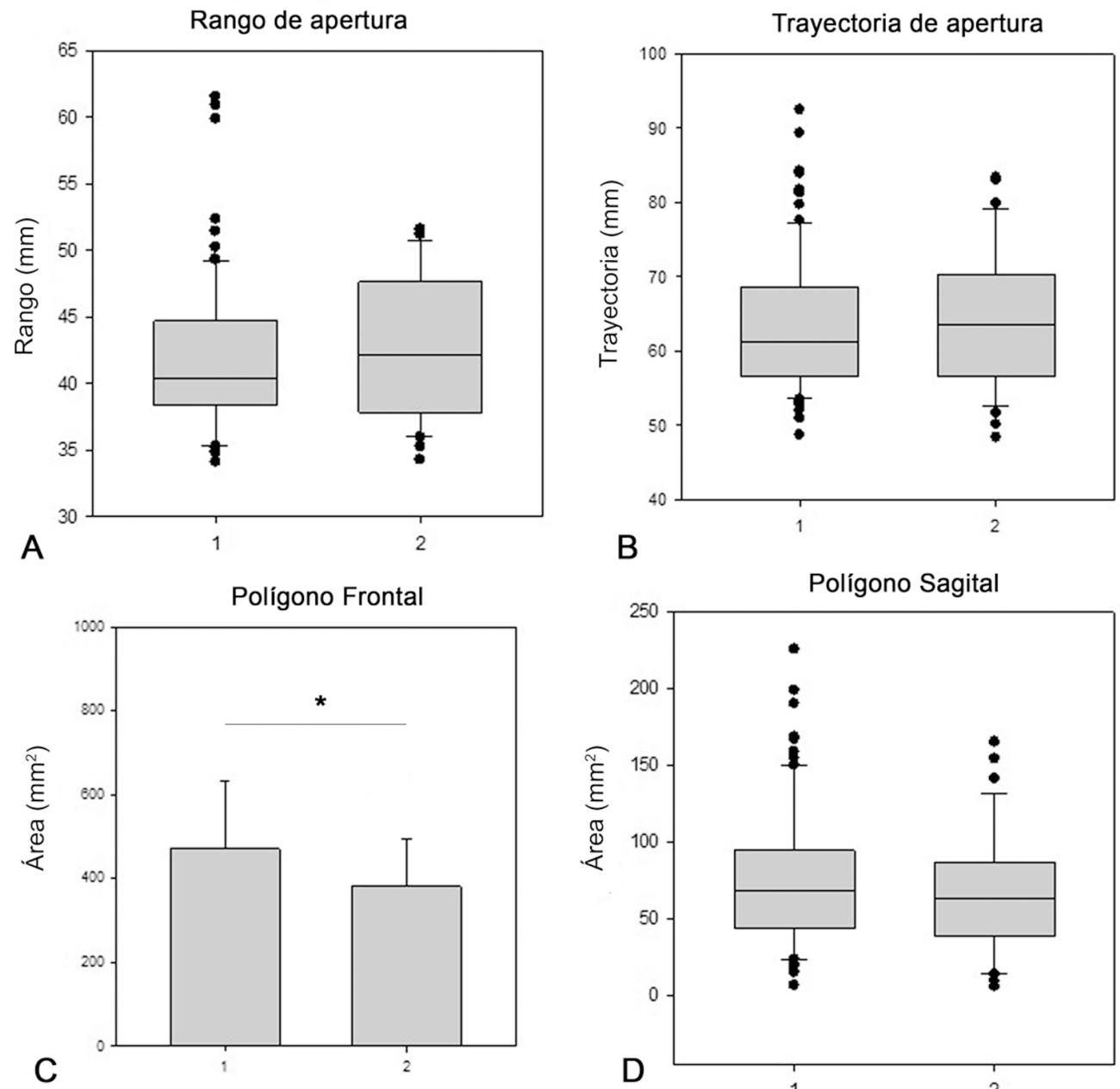

Fig. 2. Comparación del rango de apertura $(A)$, trayectoria de apertura $(B)$, área del polígono de Posselt en el plano frontal $(C)$ y área del polígono de Posselt en el plano sagital (D) entre aquellos sujetos que requieren una evaluación más exhaustiva para el diagnóstico de TTM (2) y aquellos sujetos que no la requieren (1). El signo * indica diferencia significativa $(p=0,003)$.

Al comparar los valores de la trayectoria de apertura (Fig. 3), se encontró que los hombres que respondieron "no" a las preguntas 1 ( ¿Tiene usted dificultad o dolor al abrir su boca, por ejemplo, al bostezar?) y 2 (¿Siente que su mandíbula se le "atasca", se le "traba" o se le "sale"?) su trayectoria fue mayor respecto de las mujeres que respondieron "no" a las mismas preguntas ( $p<0,05$; Fig. $3 A$ y $B)$. En tanto, la trayectoria de los hombres que respondieron "no" a la pregunta 5 (¿Siente regularmente sus mandíbulas rígidas, apretadas (agarrotadas), cansadas?) fue ma- yor respecto a las mujeres que respondieron "no" y a otros hombres que respondieron "si" presentar el síntoma ( $p<0,05$ y $p<0,05$ respectivamente; Fig. $3 C$ ). Finalmente, la trayectoria de los hombres que respondieron "no" a la pregunta 7 ( $¿$ Tiene frecuentes dolores de cabeza o dolor de cuello o de nuca?) fue mayor respecto de las mujeres que respondieron "no" ( $p$ $<0,05$; Fig. 2D), y asimismo la trayectoria de mujeres que respondieron "si" fue mayor a la de otras mujeres que respondieron "no" presentar el síntoma $(p<0,05$; Fig. 3D). 

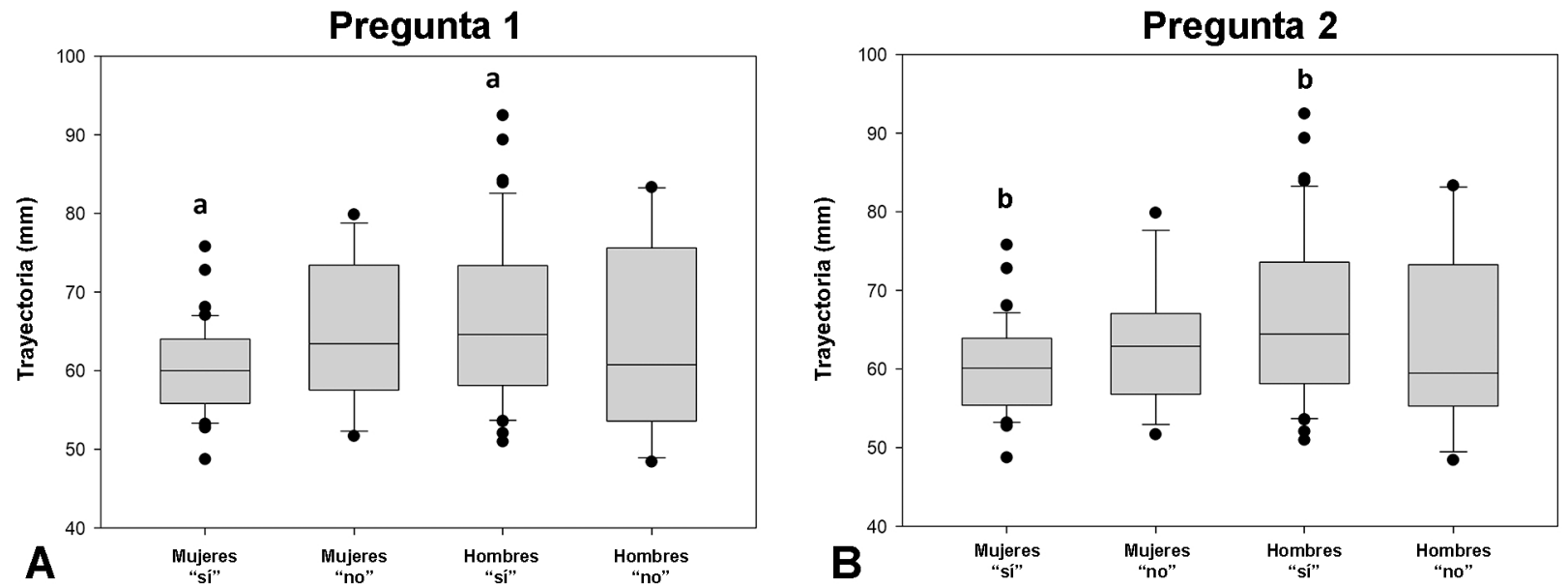

Pregunta 5
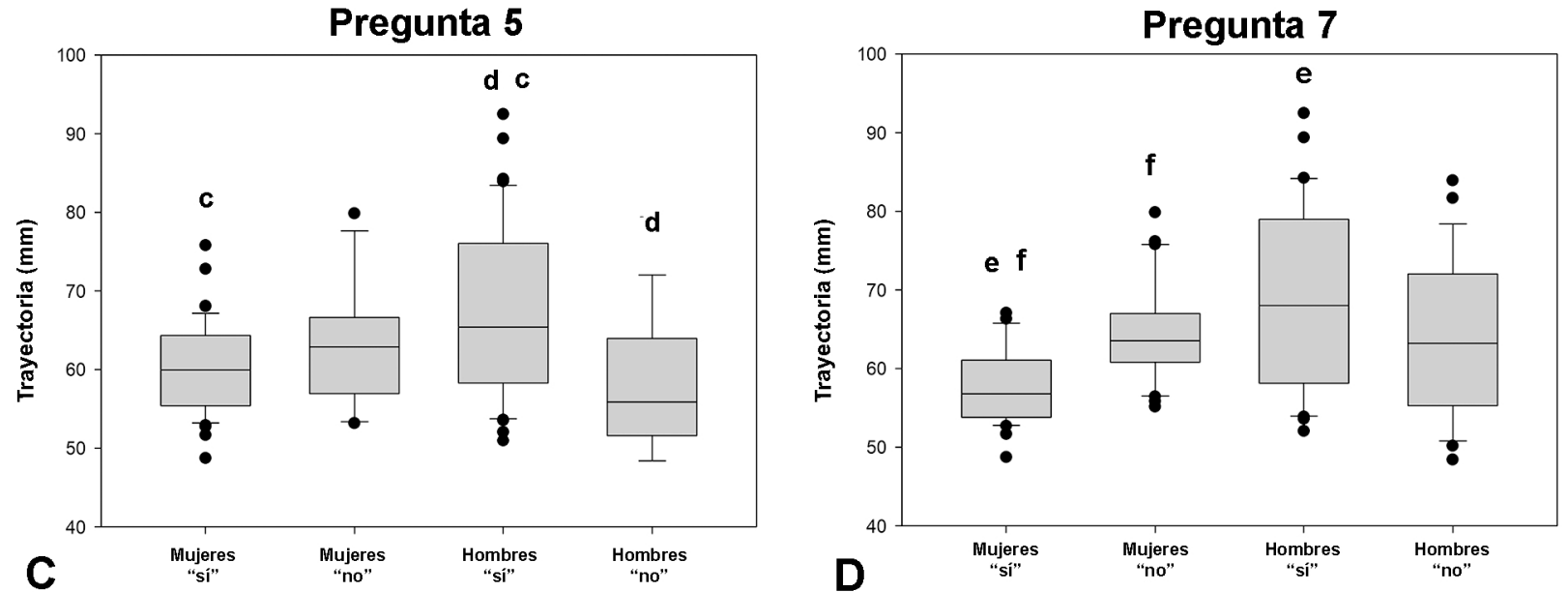

Fig. 3. Comparación de la trayectoria de apertura entre hombres y mujeres agrupados según sus respuestas ("sí" o "no") a las preguntas 1 (¿Tiene usted dificultad o dolor al abrir su boca, por ejemplo, al bostezar?), 2 (¿Siente que su mandíbula se le "atasca", se le "traba" o se le "sale"?), 5 (¿Siente regularmente sus mandíbulas rígidas, apretadas (agarrotadas), cansadas?) y 7 (¿Tiene frecuentes dolores de cabeza o dolor de cuello o de nuca?). Las letras de la "a" a la "f" indican diferencias significativas.

Pregunta 1

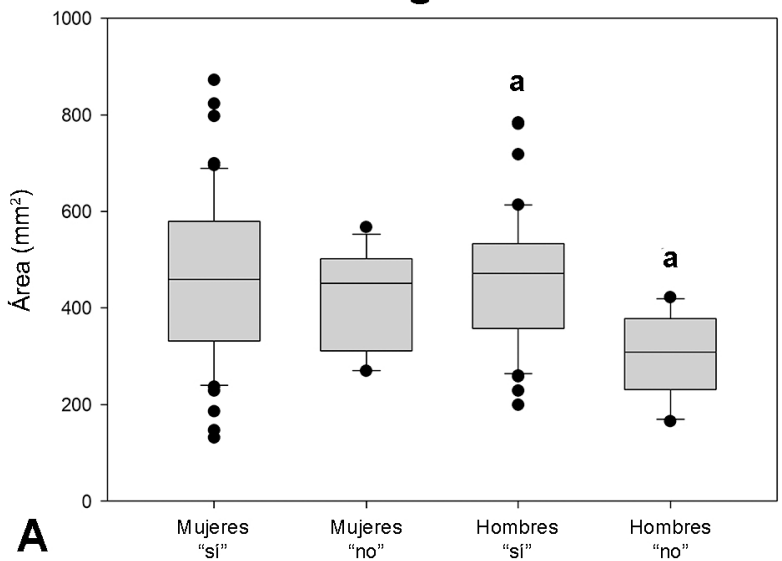

Pregunta 4

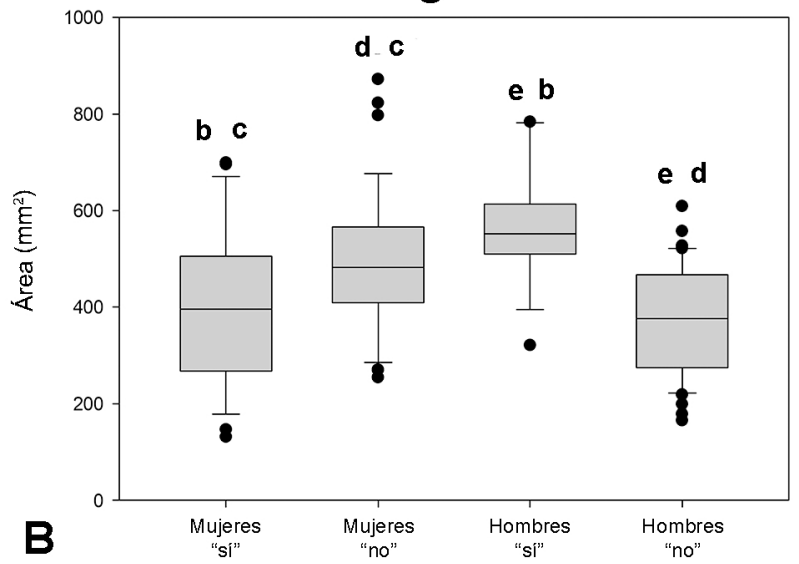

Fig. 4. Comparación del área comprendida en el polígono de Posselt en el plano frontal entre hombre y mujeres agrupados según sus respuestas ("si" o "no") a las preguntas 1 (¿Tiene usted dificultad o dolor al abrir su boca, por ejemplo, al bostezar?) y 4 (¿Ha notado ruidos en las articulaciones mandibulares?). Las letras de la "a" a la "d" indican diferencias significativas. 
Al comparar los valores del área del polígono de Posselt en el plano frontal (Fig. 4), se encontró que el área descrita por los hombres que respondieron "si" a la pregunta 1 (¿Tiene usted dificultad o dolor al abrir su boca, por ejemplo, al bostezar?) fue mayor respecto del área descrita por otros hombres que respondieron "no" presentar el síntoma ( $p<0,05$; Fig. 4A). Finalmente, se encontraron diferencias significativas en el área del polígono entre todos los grupos, hombres y mujeres que respondieron sí o no a la pregunta $4(¿ \mathrm{Ha}$ notado ruidos en las articulaciones mandibulares?) (Fig. 4B).

\section{DISCUSIÓN}

Muchos estudios mencionan que la limitación del movimiento mandibular es uno de los principales signos encontrados en pacientes con TTM (McNeill et al., 1980; Luz et al., 1995; Leamari et al., 2019), razón por la cual su evaluación representa una invaluable herramienta para el diagnóstico de problemas en el sistema masticatorio. La comprensión de la dinámica mandibular posee gran relevancia en la práctica clínica, pues permitiría la detección temprana de algunos TTM y, por tanto, su tratamiento oportuno. En tanto, la evaluación de la presencia de síntomas, también es un buen indicador para el clínico ante la sospecha de TTM en los pacientes, sin embargo, su relación con el movimiento mandibular aun no es del todo clara. Por esta razón en nuestro estudio hemos relacionado la prevalencia de síntomas asociados a TTM con el análisis exhaustivo del movimiento mandibular, con el objetivo de establecer si la presencia de algunos síntomas determina o no una diferencia en la dinámica mandibular.

La prevalencia de padecer algún síntoma relacionado a TTM es alto y varía de acuerdo a las características demográficas de la población (Johansson et al., 2006). Por ejemplo, se ha reportado que varios signos y síntomas relacionados con TTM aumentan con la edad (Köhler et al., 2009), o que en la población adulta las mujeres presentan con mayor frecuencia dolor o sonidos en la ATM, dientes sensibles o síntomas de boca urente (Johansson et al., 2003). En nuestro estudio, hemos encontrado que al menos el $85 \%$ de los participantes relata presentar algún tipo de síntoma relacionado con TTM, lo cual se contrapone a la prevalencia que reportan algunos estudios tales como el de Karthik et al. (2017) quienes reportan un $23 \%$, o el de Karibe et al. (2015) quienes reportan un $12,9 \%$. Estas diferencias, sin embargo, deben ser analizadas con precaución debido a las diferencias notables entre los tamaños de las muestras analizadas (y sus modos de selección) y los instrumentos de evaluación aplicados.

En nuestro estudio hemos evaluado el autoreporte de síntomas de TTM en un grupo de sujetos jóvenes aplicando un cuestionario clásico, breve y sencillo, que es parte de un examen de screening para la búsqueda de TTM en los pacientes (Delcanho). Este examen de screening también consta de una examinación clínica, cuyo análisis se ha obviado en este reporte debido a consideraciones metodológicas realizadas por expertos y a limitaciones propias del proceso de evaluación de participantes. De este modo, nuestra interrogante de investigación se ha centrado en la comparación cuantitativa del movimiento mandibular entre sujetos que relatan algún síntoma asociado a TTM y aquellos que se presentan asintomáticos. Nuestro primer hallazgo es que los participantes que requieren una evaluación más exhaustiva para la búsqueda de TTM (clasificados según sus respuestas al cuestionario) presentan una limitación del movimiento mandibular, evidenciado a través del área del polígono de Posselt que describen sus mandíbulas en el plano frontal, lo cual reafirma lo descrito por Celic' et al. (2003) y Mazzetto et al. (2017).

En un análisis más detallado, en nuestro estudio encontramos que los valores de la trayectoria de apertura mandibular (recorrido que realiza la mandíbula, diferente al valor de desplazamiento total entre PMI y punto de apertura máxima) varían según de que síntoma se trate. La trayectoria de apertura en hombres que presentan "mandíbulas rígidas, apretadas o cansadas" es significativamente menor al de hombres sin ese síntoma, en tanto, la trayectoria de mujeres que presentan "dolores de cabeza, cuello o nuca" es significativamente mayor al de mujeres sin ese síntoma. Por otro lado, el área del polígono de Posselt descrito por los hombres, en el plano frontal y que presentaban "dificultad para abrir la boca", fue significativamente mayor al de hombres sin ese síntoma. Finalmente, nuestros resultados indican que el "ruido en las articulaciones mandibulares" es un determinante en el valor del área del mismo polígono, siendo significativamente menor en mujeres sin ese síntoma respecto de aquellas que no lo presentaron, y siendo significativamente mayor en hombres que sí presentaron ese síntoma respecto de aquellos que no lo presentaron. 


\section{CONCLUSIONES}

En este estudio hemos comprobado que algunos síntomas asociados a TTM podrían significar clínicamente una diferencia en las amplitudes del movimiento mandibular descrito por los pacientes. Los principales síntomas que afectarían los valores de la trayectoria de apertura son la presencia de "mandíbulas rígidas, apretadas o cansadas" y los "dolores de cabeza, cuello o nuca". Por ejemplo, en nuestro estudio la media de la trayectoria de apertura mandibular en hombres con estos síntomas fue de 55,89 y 56,78 mm, respectivamente, un valor por debajo de la media encontrada en hombres que no reportaron estos síntomas $(65,41 \mathrm{~mm})$.

En tanto, los principales síntomas que afectarían los valores de los movimientos bordeantes (que describen el polígono de Posselt en el plano frontal) son la "dificultad para abrir la boca" y el "ruido en las articulaciones mandibulares". Por ejemplo, en nuestro estudio el promedio del área del polígono en hombres con estos síntomas fue de $462,29 \pm 138,37 \mathrm{~mm}^{2}$, un valor por sobre el promedio encontrado en hombres que no reportaron estos síntomas $\left(303,22 \pm 85,73 \mathrm{~mm}^{2}\right)$. En el caso de los participantes que al responder el cuestionario de auto reporte, arrojaron la necesidad de una evaluación por un especialista, el área promedio $\left(381,21 \pm 111,12 \mathrm{~mm}^{2}\right)$ del movimiento bordeante en el plano frontal, fue significativamente menor $\left(470,91 \pm 160,85 \mathrm{~mm}^{2}\right)$ que aquellos que no requirieron una evaluación más exhaustiva, evidenciándose la limitación de este movimiento, en los sujetos con posible TTM.

La evaluación del movimiento mandibular debe hacerse con el contexto de exámenes y evaluaciones complementarias, así como a la luz de las características constitutivas de los pacientes, ya que se ha visto que la limitación del movimiento mandibular en las mujeres no siempre ocurre ante la presencia de un síntoma. Por último, este estudio exploratorio reafirma la necesidad de contar con una evaluación más profunda y con una muestra de mayor tamaño, que correlacione la presencia de síntomas autorreportados y el análisis cuantitativo de movimiento mandibular. Un nuevo análisis debiera considerar en su metodología la utilización de instrumentos acordes a los nuevos criterios de diagnóstico para TTM.
AGRADECIMIENTOS. Financiado parcialmente por la Dirección de Investigación de la Universidad de La Frontera, Chile.

ARIAS, A.; SARAVIA, D.; QUINTANA, M.; DIAS, F.; LEZCANO, M. F.; FARFÁN, C. \& FUENTES, R. Relationship between symptoms of self-reported temporomandibular disorders and mandibular movement of young adult subjects. Int. J. Odontostomat., 13 (4):466474, 2019.

ABSTRACT: Both the limitation of mandibular movement and the self-report of symptoms of temporomandibular disorders (TMD) are used for the diagnosis of patients in clinical diagnosis. However, the relationship between the symptoms and mandibular movement is not entirely clear. Therefore, the objective of this study was to relate the presence of symptoms associated with TMD with mandibular movement in a sample of young and healthy adult subjects. This exploratory study involved 40 medically healthy dentistry students with a continuous and complete maxillary and mandibular arch (up to the first molar). Each subject was asked to answer a self-report questionnaire for symptoms associated with TTM recommended by the American Academy of Orofacial Pain (AAOP). We also recorded and analyzed their mandibular movements using 3D electromagnetic articulography, with the aim of correlating the presence of symptoms with the range and trajectory of opening, and the area included in what is described as a polygon of Posselt in the frontal and sagittal plane. No significant differences were found between the answers to each question between men and women. According to the responses and to Delcanho, 12 participants turned out to need a more exhaustive evaluation. In this group it was found that the area of the Posselt polygon in the frontal plane was significantly smaller than that of those participants who did not require a more extensive evaluation $(p=0.003)$. The values of the mandibular opening path vary depending on the symptom involved. The main symptoms that would affect the values of the bordering movements are the "difficulty to open the mouth" and "noise in the mandibular joints". With this study, we have shown that the limitation of mandibular movement as a sign of TMD should be assessed with caution, as, according to the presence of certain symptoms and other characteristics of subjects (such as sex), its value may vary.

KEY WORDS: temporomandibular disorders, mandibular movement, electromagnetic articulography.

\section{REFERENCIAS BIBLIOGRÁFICAS}

Al-Dlaigan, Y. H. \& Asiry, M. A. Maximum mouth opening in Saudi adolescents. J. Int. Oral Health, 6(6):45-9, 2014. 
Aravena, P. C.; Arias, R.; Aravena-Torres, R. \& Seguel-Galdames, F. Prevalencia de trastornos temporomandibulares en adolescentes del Sur de Chile, año 2015. Rev. Clin. Periodoncia Implantol. Rehabil. Oral, 9(3):244-52, 2016.

Casanova-Rosado, J. F.; Medina-Solís, C. E.; Vallejos-Sánchez, A. A.; Casanova-Rosado, A. J.; Hernández-Prado, B. \& Avila-Burgos, L. Prevalence and associated factors for temporomandibular disorders in a group of Mexican adolescents and youth adults. Clin. Oral Investig., 10(1):42-9, 2006.

Celic, R.; Jerolimov, V.; Knezovic Zlataric, D. \& Klaic, B. Measurement of mandibular movements in patients with temporomandibular disorders and in asymptomatic subjects. Coll. Antropol., 27 Suppl. 2:43-9, 2003.

Delcanho, R. Screening for temporomandibular disorders in dental practice. Aust. Dent. J., 39(4):222-7, 1994.

Fuentes, R.; Dias, F.; Álvarez, G.; Lezcano, M. F.; Farfán, C.; Astete, N. \& Arias, A. Application of 3D electromagnetic articulography in dentistry: mastication and deglutition analysis. protocol report. Int. J. Odontostomat., 12(1):105-12, 2018.

Granger, E. R. Functional relations of the stomatognathic system. J. Am. Dent. Assoc., 48(6):638-47, 1954.

Johansson, A.; Unell, L.; Carlsson, G. E.; Söderfeldt, B. \& Halling, A. Gender difference in symptoms related to temporomandibular disorders in a population of 50 -year-old subjects. J. Orofac. Pain, 17(1):29-35, 2003.

Johansson, A.; Unell, L.; Carlsson, G. E.; Söderfeldt, B. \& Halling, A. Risk factors associated with symptoms of temporomandibular disorders in a population of 50- and 60-year-old subjects. J. Oral Rehabil., 33(7):473-81, 2006.

Karibe, H.; Shimazu, K.; Okamoto, A.; Kawakami, T.; Kato, Y. \& Warita-Naoi, S. Prevalence and association of self-reported anxiety, pain, and oral parafunctional habits with temporomandibular disorders in Japanese children and adolescents: a cross-sectional survey. B. M. C. Oral Health, 15:8, 2015.

Karthik, R.; Hafila, M. I. F.; Saravanan, C.; Vivek, N.; Priyadarsini, P. \& Ashwath, B. Assessing prevalence of temporomandibular disorders among university students: a questionnaire study. J. Int. Soc. Prev. Community Dent., 7(Suppl. 1):S24-S29, 2017.

Klasser, G. D. \& Greene, C. S. Oral appliances in the management of temporomandibular disorders. Oral Surg. Oral Med. Oral Pathol. Oral Radiol. Endod., 107(2):212-23, 2009.

Köhler, A. A.; Helkimo, A. N.; Magnusson, T. \& Hugoson, A. Prevalence of symptoms and signs indicative of temporomandibular disorders in children and adolescents. A cross-sectional epidemiological investigation covering two decades. Eur. Arch. Paediatr. Dent., 10 Suppl. 1:16-25, 2009.

Leamari, V. M.; Rodrigues, A. F.; Camino Junior, R. \& Luz, J. G. C. Correlations between the Helkimo indices and the maximal mandibular excursion capacities of patients with temporomandibular joint disorders. J. Bodyw. Mov. Ther., 23(1):148-52, 2019.

Li, X. Y.; Jia, C. \& Zhang, Z. C. The normal range of maximum mouth opening and its correlation with height or weight in the young adult Chinese population. J. Dent. Sci., 12(1):56-9, 2017.

Luz, J. G.; Uono, H. H. \& Yamamoto, M. K. Passive mandibular border positions and their relationships with signs and symptoms of craniomandibular disorder. A study of linear and angular measurements. J. Nihon Univ. Sch. Dent., 37(1):28-32, 1995.

Manfredini, D.; Guarda-Nardini, L.; Winocur, E.; Piccotti, F.; Ahlberg, J. \& Lobbezoo, F. Research diagnostic criteria for temporomandibular disorders: a systematic review of axis I epidemiologic findings. Oral Surg. Oral Med. Oral Pathol. Oral Radiol. Endod., 112(4):453-62, 2011.

Manns Freese, A. Sistema Estomatognático. Bases Biológicas y Correlaciones Clínicas. Santiago de Chile, Ripano, 2011.
Mazzetto, M. O.; Anacleto, M. A.; Rodrigues, C. A.; Bragança, R. M.; Paiva, G. \& Valencise Magri, L. Comparison of mandibular movements in TMD by means of a 3D ultrasonic system and digital caliper rule. Cranio, 35(1):46-51, 2017.

McNeill, C. Temporomandibular Disorders: Guidelines for Classification, Assessment and Management. $2^{\mathrm{a}}$ ed. Chicago, Quintessence, 1993.

McNeill, C.; Danzig, W. M.; Farrar, W. B.; Gelb, H.; Lerman, M. D.; Moffett, B. C.; Pertes, R.; Solberg, W. K. \& Weinberg, L. A. Position paper of the American Academy of Craniomandibular Disorders. Craniomandibular (TMJ) disorders--the state of the art. J. Prosthet. Dent., 44(4):434-7, 1980.

Paulino, M. R.; Moreira, V. G.; Lemos, G. A.; Silva, P. L. P. D.; Bonan, P. R. F. \& Batista, A. U. D. Prevalence of signs and symptoms of temporomandibular disorders in college preparatory students: associations with emotional factors, parafunctional habits, and impact on quality of life. Cien. Saude Colet., 23(1):173-86, 2018.

Pereira, L. J.; Pereira-Cenci, T.; Pereira, S. M.; Cury, A. A.; Ambrosano, G. M.; Pereira, A. C. \& Gavião, M. B. Psychological factors and the incidence of temporomandibular disorders in early adolescence. Braz. Oral Res., 23(2):155-60, 2009.

Ramirez Aristeguieta, L. M.; Sandoval Ortiz, G. P. \& Ballesteros, L. E. Theories on otic symptoms in temporomandibular disorders: past and present. Int. J. Morphol., 23(2):141-56, 2005.

Sena, M. F.; Mesquita, K. S.; Santos, F. R.; Silva, F. W. \& Serrano, K. V. Prevalence of temporomandibular dysfunction in children and adolescents. Rev. Paul. Pediatr., 31(4):538-45, 2013.

Taboada, A. O.; Gómez, G. Y. L.; Taboada, A. S. \& Mendoza, N. V. M. Prevalencia de signos y síntomas de los trastornos temporomandibulares en un grupo de adultos mayores. Rev. Asoc. Dent. Mex., 61(4):125-9, 2004.

Wu, N. \& Hirsch, C. Temporomandibular disorders in German and Chinese adolescents. J. Orofac. Orthop., 71(3):187-98, 2010.

Dirección para correspondencia:

Prof. Dr. Ramón Fuentes Fernández

Research Centre for Dental Sciences

Dental School, Universidad de La Frontera

Av. Francisco Salazar 01145

Temuco

CHILE

Email: ramon.fuentes@ufrontera.cl

Recibido : 10-05-2019

Aceptado: 11-07-2019 